\title{
How vegetative is the vegetative state? Preserved semantic processing in VS patients - Evidence from N 400 event-related potentials ${ }^{1}$
}

\author{
Paul W. Schoenle* and W. Witzke \\ University of Konstanz and Neurological Rehabilitation Center NRC, Gustav-Ricker-Str. 4, D-39102 Magdeburg, \\ Germany \\ Tel.: +49 391610 1101; Fax: +49 391610 1166; E-mail: paul.schoenle@uni-konstanz.de
}

\begin{abstract}
Event-related potentials (ERPs) can provide valuable information about cognitive capabilities in severely braindamaged patients. This study examined 120 patients with severe brain damage using event related potentials ERPs (N 400) to gain information about their remaining semantic processing capabilities and to contribute to differential diagnosis. Patients were classified into three diagnostic groups: patients in vegetative state (VS), patients in near vegetative state (NEVS) and patients not in vegetative state (NOVS). N400 ERPs were analyzed on an individual basis. All three groups could be differentiated by N400. While VS-patients as a group were least likely to show N400, some VS-patients (approx. 12\%) showed clear semantic N400 potentials as an indication of semantic processing capabilities. Patients in NEVS showed significantly more intact semantic capabilities (76.74\%) than the VS-group despite little clinical differences between the two groups. Thus, ERPs provide valuable information about patients with brain injury whose clinical conditions often do not allow a true assessment of their cognitive capabilities. Given these findings, we would espouse that both ethical and legal debate should take into account results of ERP studies of such patients.
\end{abstract}

Keywords: N400, semantic processing, vegetative state, minimally conscious state, severe brain injury

\section{Introduction}

As expressed in the original definition of VS [1], there have been commonly accepted beliefs in the medical community about the diagnostic criteria of the vegetative state (VS) syndrome. Several consensus statement papers and guidelines on management of VS [27] have confirmed them. The criteria include intact sleep-wake cycles, completely or partially preserved hypothalamic and brain stem autonomic functions in absence of sustained, reproducible, purposeful, or vol-

\footnotetext{
${ }^{*}$ Corresponding author.
}

${ }^{1}$ See also [14]. untary behavioral responses to visual, auditory, tactile, or noxious stimuli, and complete unawareness of the self and the environment. The criteria also include a lack of evidence for mental activity, cognition, as well as, language comprehension or expression.

When we opened a special rehabilitation unit for patients in VS in 1991, we introduced the competence versus performance distinction from linguistic theory [8] and the covert versus overt behavior notion from psychophysiology [9] to re-conceptualize the VS syndrome. Competence is a speaker's tacit internal knowledge of language, while performance refers to the usage of language in concrete situations: it is the application and externalization of the otherwise hidden linguistic capabilities during speech. Likewise overt 


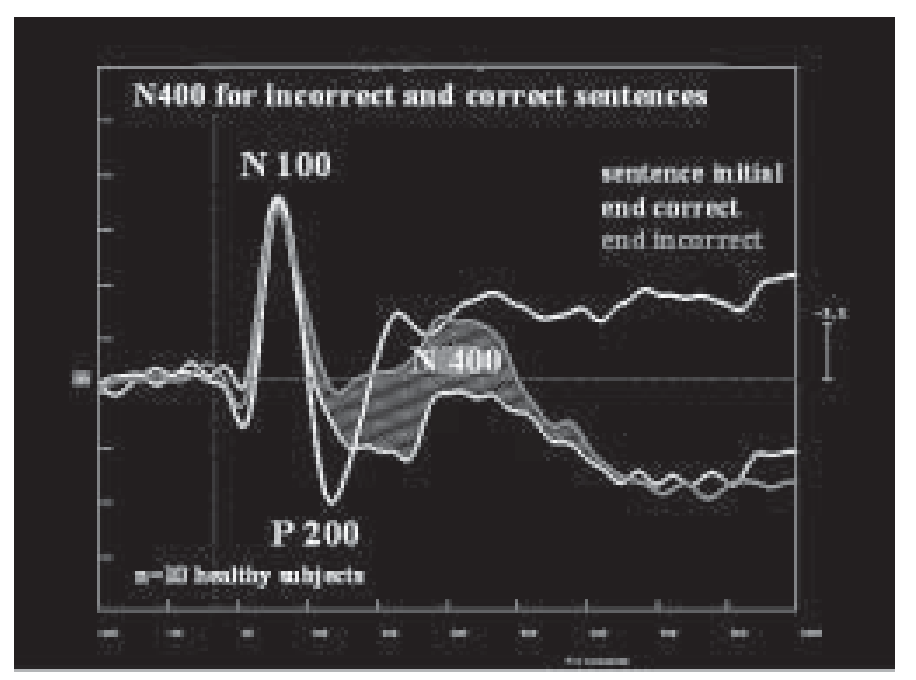

Fig. 1. N400 event-related potential in normal subjects. A N400 wave (grey dashed area) is recorded in healthy subjects when they hear semantically anomalous sentences ("The coffee is to hot to fly.") vs. regular sentences ("The coffee is to hot to drink.”) (lower white curve). Site of recording is $\mathrm{Cz}$, y-axis indicates amplitude ( $3.5 \mu \mathrm{V}$ per unit) with negativity plotted upward. $\mathrm{Y}$ axis represents time (120 ms per unit).

behavior is the externally observable activity of a person in contrast to covert behavior, which denotes behavior going on inside a person and is only observable with the aid of instruments. Transferring these concepts to behavioral phenomena after brain damage, we have to distinguish the loss of internal knowledge and processing capabilities from a problem with their externalization in the moment of performance.

Thus, the question arises whether patients in VS suffer "only" from performance problems or whether they have lost their inner knowledge and processing capabilities in the various mental and cognitive domains. For example, if patients in VS were able to process language, this would be strong evidence for preserved higher cognitive capabilities and an argument against the implicit assumption that VS patients only function at a vegetative level.

In this study, we used possibly existing linguistic capabilities to test for the existence of cognitive capabilities in VS patients. We chose testing linguistic faculties because language is the most common and complex human cognitive system. Moreover, it can easily be tested in a clinical setting using the N-400 paradigm [10,11]. The N-400 is an event-related potential with a negative deflection around $400 \mathrm{~ms}$, and is found in healthy subjects when they see or hear semantically anomalous sentences like "The coffee is too hot to fly" versus "The coffee is too hot to drink” (Fig. 1). We hypothesized that at least some patients diagnosed as being in a vegetative state would show the N-400 phenomenon and, therefore, would not be exclusively vegetative.

\section{Method}

\subsection{Patients}

We examined 120 severely brain damaged patients in the course of the standard clinical neurophysiologic routine program for patients entering our special treatment unit for VS patients (Table 1 ). $70 \%$ were male, the mean age was 44.2 years (SD 14.7) ranging from 18 to 75 years. $41.7 \%$ suffered from traumatic brain injury, 25.8\% from hypoxic brain damage and 32.5\% from CVA. Patients comprised three categories: patients in vegetative state (VS-patients, $n=35.8 \%$ ), in near vegetative state (NEVS, 19.2\%) and being severely brain damaged but not in VS (NOVS, $n=45 \%$ ). VS patients were classified according to conventional clinical criteria (see above). In addition, we applied criteria such as habituation, eye fixation, visual pursuit, and orienting reactions. If one occurred without other signs of meaningful behavior we classified patients as NEVS. If patients showed some meaningful behavior, we classified them as NOVS (neither VS nor NEVS).

The habituation paradigm tests blink responses in the visual (blink to thread), acoustic (toy frog producing a sudden noise with $90 \mathrm{~dB}$ at patients' ears) and tactile domains (slight reflex hammer taps on the glabella) with 10 stimuli in each modality, one stimulus applied per second. Patients in VS show blink responses without inhibition (10 responses per 10 stimuli) while patients out of VS habituate (e.g. V6/10, A5/10, T8/10), eventually improving to normal immediate habituation 
Table 1

Overview on the diagnostic routine program of neurophysiologic tests in our clinical setting for investigation of patients in VS and related states

\begin{tabular}{|c|c|c|}
\hline Procedure & Parameters & Clinical objectives \\
\hline Longterm-EEG & $\begin{array}{l}\text { Frequency spectra, frequency peaks, relative } \\
\text { power per band, variance of amplitude }\end{array}$ & $\begin{array}{l}\text { State of conciousness fluctuation of vigilance; sleep/wake } \\
\text { rhythm, epileptic activities }\end{array}$ \\
\hline Event-related potentials & $\begin{array}{l}\text { Amplitude and latency of components: } \\
\text { - N100 } \\
\text { - Mismatch negativity } \\
\text { - Processing negativity } \\
\text { - P300 } \\
\text { - N400 } \\
\text { - Slow wave } \\
\text { - CNV }\end{array}$ & $\begin{array}{l}\text { Cognitive functions: Consciousness, attention, perception, } \\
\text { memory, language, learning } \\
\text { Automatic detection, selective filtering } \\
\text { Automatic discrimination } \\
\text { Selective processing } \\
\text { Controlled processing of stimuli } \\
\text { Semantic processing of verbal material } \\
\text { Long lasting processing } \\
\text { Attention control }\end{array}$ \\
\hline Blink reflex & Amplitude & Habituation \\
\hline EEG- and ERP-Mapping & Amplitude maps over time or frequency & Topography, localization \\
\hline
\end{tabular}

like in NOVS patients and healthy subjects $(1 / 10,2 / 10$ in any modality) [12]. All our patients had normal early acoustic evoked potentials and acoustic blink reflexes indicating intact primary auditory pathways while they had abnormal EEG with slowing in delta and slow theta bands. The first finding implies that using acoustic stimuli to test linguistic capabilities was a valid method.

The NEVS category was introduced and operationally defined (see above) for this study in 1994 before the concept of minimally conscious state (MCS) was introduced [13]. According to the definition of MCS, patients in MCS appear to be in a somewhat higher functional state than NEVS patients. Unlike MCS patients, NEVS patients were not able to follow simple commands, produce gestural or verbal yes/no responses, intelligible verbalization or purposeful behavior. This difference is important to note when interpreting the N400 results.

\subsection{Material and procedure}

We prepared 200 sentences consisting of 5 words each. A set of 100 sentences had a semantically incorrect final word ("The coffee is to hot to fly"), and the control set of 100 sentences ended with a semantically correct word ("the coffee is too hot to drink"). The 200 sentences were randomized and acoustically presented to all patients through earphones (Sennheiser HD 55) using Neurostim (Neuroscan $\left.{ }^{\circledR}\right)$. Computerized EEG was recorded and averaged. N400s were visually identified by three independent investigators. Results were accepted as valid when the evaluations of all investigators agreed. All investigators were experienced neurophysiologists. Shapes of waves were classified into three categories: no N400 present, emerging N400 (see A1 in Fig. 2), clear N400 deflection (see A2 in Fig. 2), and N400 with an amplitude $\geqslant 10 \mu \mathrm{V}$.

\section{Results and discussion}

As expected, patients who were not in VS (NOVS) produced the most robust results on N-400 testing (Fig. 3). Only 9.23\% of patients in NOVS did not show a N-400, while $90.17 \%$ were able to distinguish semantically correct from semantically anomalous sentences. Patients in near vegetative state (NEVS) exhibited no $\mathrm{N}-400$ in $23.26 \%$ of cases. However, $76.74 \%$ produced a $\mathrm{N}-400$ in one of the three forms (51.36\%, 10.69\%, $14.69 \%)$. While patients in VS were lacking a N-400 in $61.12 \%$ of all cases, $11.96 \%$ of this patient group exhibited distinct semantic capabilities and $26.91 \%$ showed “emerging” N-400 waves. In total, $38.87 \%$ of patients in VS showed some form of N-400 waves, implying that semantic capacities existed.

These results demonstrate that more than one third of our study group who were clinically diagnosed as being in a vegetative state were able to perform semantic analysis on acoustically presented, linguistic material. Arguing more conservatively, and not taking into account emerging $\mathrm{N}-400$ s, the brains of at least $12 \%$ of VS patients appear to be "semantically intact". This result challenges the traditional concept of VS and the primarily clinical diagnostic approach utilized to determine the neurological state of any low level neurological patient following acquired brain injury. We can no longer conceptualize the vegetative state as a state in which only vegetative (i.e. autonomous) functions are preserved and cognitive abilities are nil. Some VS patients, an even higher percentage of NEVS and almost all NOVS patients are able to perform semantic processing. This points to the existence of a continuum of functional states in low level neurological patients following severe acquired brain injury. It seems to be less likely that there are distinct categorical states that can be referred to by static diagnostic labels. Rather, patients 

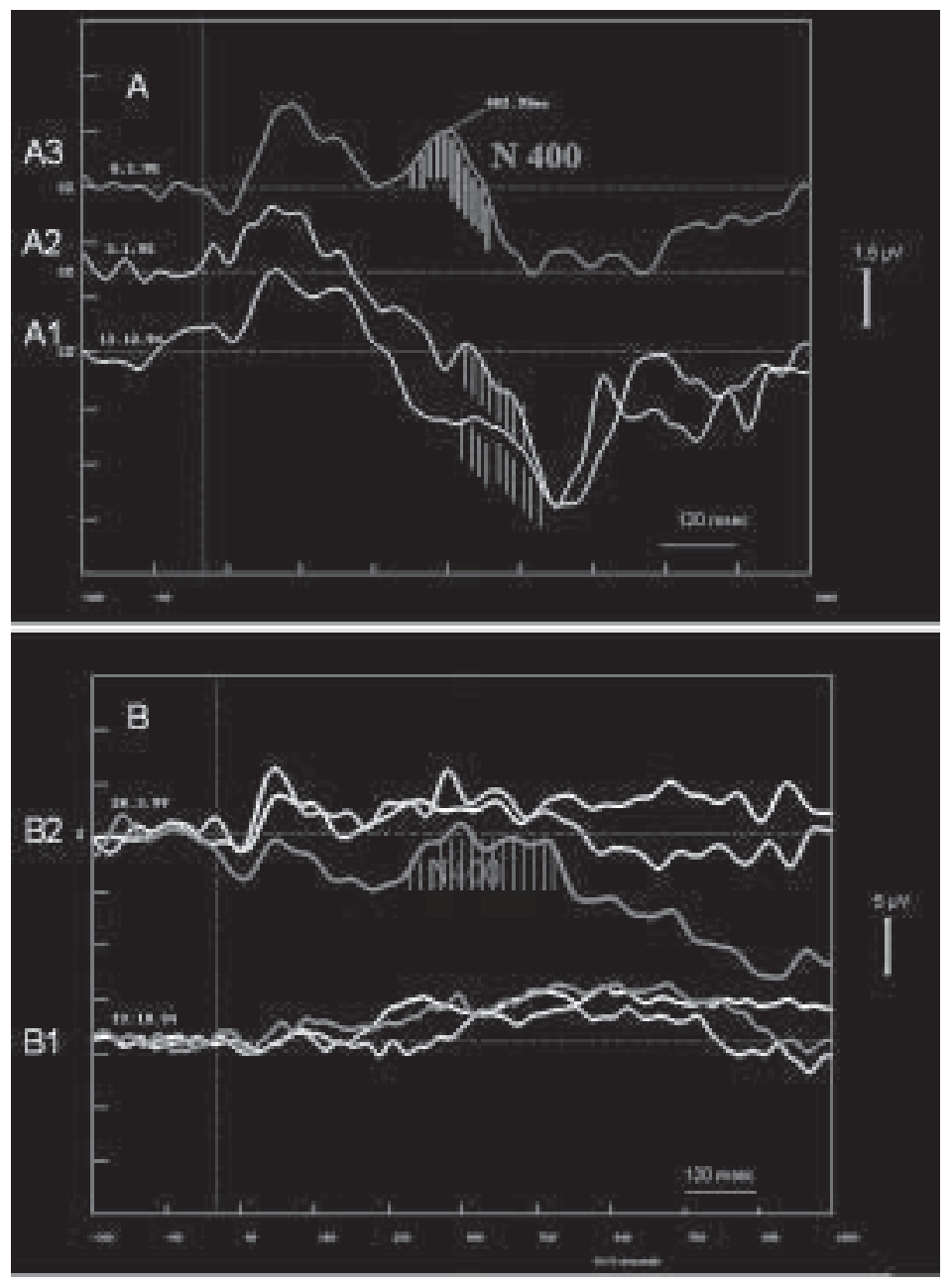

Fig. 2. Development of N400 over time. In part B (lower half) N400 developed over 17 months in a patient with postanoxic VS. At B1 no distinct processing occurred for semantically anomalous (grey) versus correct (lower white) sentences (upper white: sentence initial). After 17 months a N100/P200 (asterix) and a broad N400 (dashed area) waves could be recorded. Two asterixes in B1 indicate an emerging N100/P200. In part A (upper half) N400 develops over 26 days in a patient with VS, 6.5 months after traumatic brain injury. A: The shaded area in A1 marks the beginning of negative deflection in the N400 region in the first recording. B: The shaded area in A2 indicates a clearly delineated negative deflection (day 21) and the shaded area in A3 shows the fully developed N400 (day 26). Site of recording was $\mathrm{Cz}, \mathrm{y}$-axis indicates amplitude (1.5 $\mu \mathrm{V}$ per unit in $\mathrm{A}$ and $5 \mu \mathrm{V}$ in $\mathrm{B}$ ) with negativity plotted upward. $\mathrm{X}$ axis represents time (120 ms per unit in $\mathrm{A}$ and $\mathrm{B}$ ).

with severe brain damage appear to possess preserved internal capabilities in the sense of the covert behavior defined above. These internal functional abilities may simply not surface to the overt phenomenological performance level. Thus, it is not easy to observe them clinically, if at all.

In this covert/overt and competence/performance conceptualization of VS, a lack of response can be interpreted in a twofold manner. First, a lack of response may be due to a performance problem, i.e. a motor output deficit combined with an intact internal covert response, in our case, intact semantic processing. Second, a lack of response may reflect a breakdown of the inner covert processing ability (loss of competence). Further, both deficits may be combined in the most severe cases.

In addition, one should extend the above conceptualization of VS on a dynamic scale. It may well be that the level of functioning of the neural substrates which underlie competence and covert behavior is not "all or none" or the same all the time. When we do not find a $\mathrm{N}-400$ at given moment, we have to take into account that the brain activity of a patient may currently be reduced but may change over time. Indeed, we have seen that the appearance of N400 is state dependent. When we track cerebral activity over time with computerized 


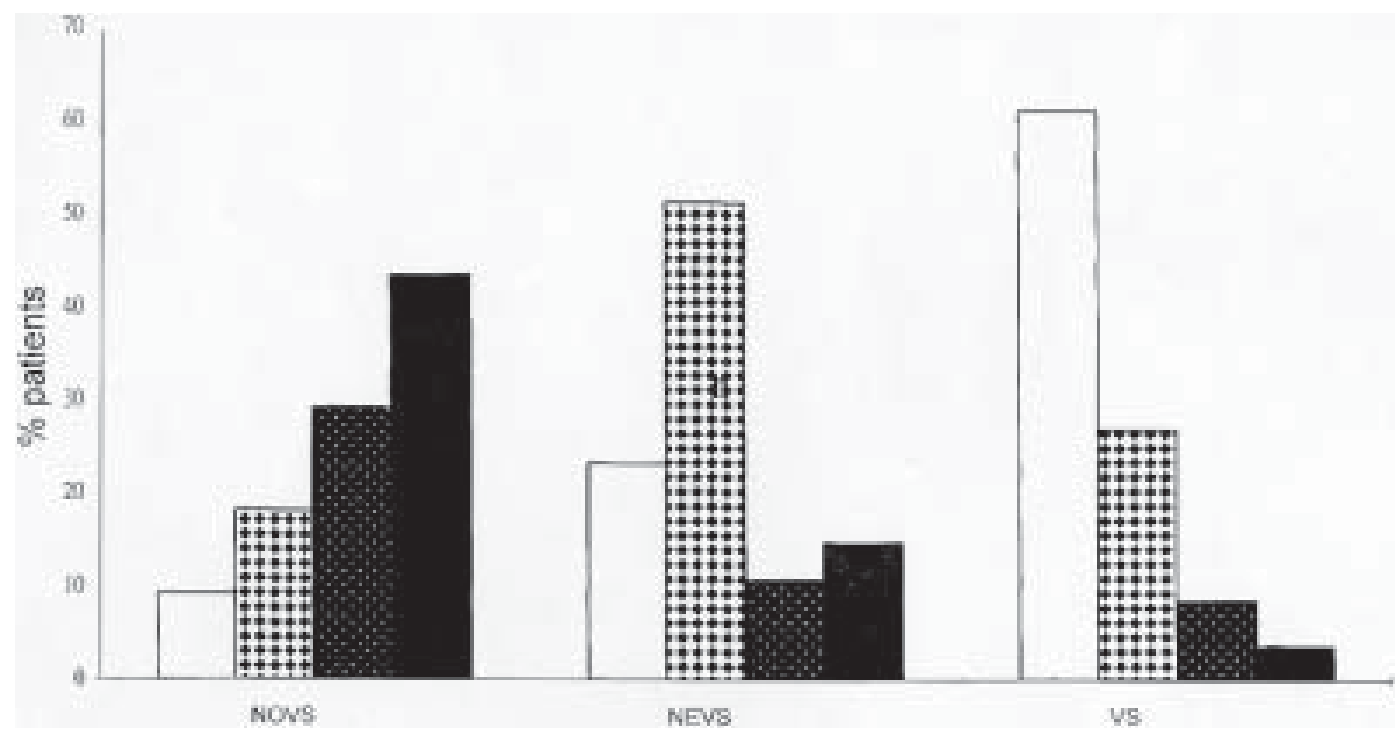

Fig. 3. N400 of severely brain damaged patients in non-VS (NOVS), near-VS (NEVS), and VS. White columns indicates no N400 present, black dots emerging N400 (see Fig. 2, A1), white dots: clearly delineated N400 (see Fig. 2, A2), black: well formed N400 (see Fig. 2, A3). In NOVS $9.23 \%$ of patients did not show a N400, the rest $90.17 \%$ did show a N400 (18.12\%, 29.17\% and 43.49\%). In NEVS 23.26\% had no N400, while $76.74 \%$ had a N400 in one of the three forms $(51.36 \%, 10.69 \mathrm{~N} 400$ could be found in $61.12 \%$ of VS patients, 38.88\% showed, however, a N400 in one of the three forms $(26.91 \%, 8.46 \%, 3.50 \%)$. While NOVS patients show the highest level of intact semantic processing, also $11.96 \%$ of VS patients clearly exhibit distinct semantic capacities and. 26.91\% show emerging N400 waves. In $61.12 \%$ cases no N400 can be found.

long term EEG, we often see free-running “sleep/wake” periods. This allows us to locate the best activity windows in the 24 to 48 hour recording time and to perform neurophysiologic tests (see Table 1) during periods of optimum brain activity.

Similarly, P-300 also turns out to be state-dependent. However, it appears, that the P-300 depends more on "overall better brain activity" than the $\mathrm{N}-400$. In some severe cases, we have observed $\mathrm{N}-400$ potentials in absence of P-300 which is likely to be due to the "unnaturalness" of tones used in the P-300 and the ecological validity of natural language applied in the N-400 paradigms [14].

Looking at long term dynamics of N-400 potentials, that is over weeks and months, and testing during optimal brain activity, we re-investigated $15 \%$ of our study group. We found improvement in $56 \%$ of the patients. Just over $38 \%$ of patients showed no change. In $0.06 \%$ of the patients, the $\mathrm{N}-400$ could not be identified even if it was present before. No major differences in N400 improvement occurred between the NEVS and VS groups. ${ }^{2}$ Hence, we cannot give a definite answer yet as to whether the N400 development has some use to further differentiate between the VS and NEVS patients.

\footnotetext{
${ }^{2}$ Most NOVS had shown N400 on first testing and were not retested.
}

We would expect NEVS patients to produce N-400s more constantly on each testing while N-400s might be more unstable in VS patients. Also faster N-400 recovery rates may be more indicative of NEVS than VS patients. Since our sample was too small, further studies should include more patients to investigate the long-term development of N-400.

The N-400 results reported here and results of other cognitive neurophysiologic tests reported earlier [14] demonstrate their clinical importance as assessment tools when analyzing and subcategorizing the functionality of patients following severe brain injury. N-400s allow tracking of distinct levels of information processing including automatic detection, discrimination, selective filtering, and controlled processing of stimuli as complex as semantically normal and anomalous sentences. N-400s also allow for "localization" of specific deficits in the functional systems of attention, perception, memory, language and learning (Table 1). Thus, $\mathrm{N}-400$ s provide a "fine tuned" picture of the functional state of these patients and give us a deeper insight into their covert inner capabilities which otherwise would likely remain unrecognized.

Whether the results of the present study are indicative of conscious behavior in VS and NEVS patients depends on the significance of semantic processing (SP) in relation to consciousness. On the one hand, SP abil- 
ities are most likely not sufficient to give rise to conscious experience. On the other, we cannot experience ourselves in a conscious way without attributing meaning to our environment and us. Thus, quite a number of patients in VS may indeed experience full or some limited form of consciousness that is otherwise not noticed clinically. To identify these patients is an important medical responsibility, especially considering the medico-legal and ethical implications of VS. Therefore, one should attempt to use neurophysiologic techniques more widely as they can be applied in a clinical setting (as on our VS unit) routinely and at relatively low cost.

Future research on neurophysiologic assessment should further detail cognitive processing capacities, extend stimulus material into other cognitive domains including executive functions and focus on emotional and affective systems.

\section{Acknowledgements}

Research reported here was supported in part by grants to PWS from Kuratorium ZNS Hannelore Kohl (1993) and from the Social Ministry of BadenWürttemberg (1992).

\section{References}

[1] B. Jennett and F. Plum, Persistent vegetative state after brain damage: a syndrome in search of a name, Lancet I (1972), 734-737.

[2] Multi-Society Task Force on PVS. Medical aspects of the persistent vegetative state (first part), N Engl J Med 330 (1994), 1499-1508.

[3] Multi-Society Task Force on PVS. Medical aspects of the persistent vegetative state (second part), $N$ Engl $J$ Med 330 (1994), 1572-1579.

[4] American Neurological Association Committee on Ethical Affairs. Persistent vegetative state: report of the American Neurological Association committee on ethical affairs, Ann Neurol 33 (1993), 386-390.

[5] BMA Medical Ethics Committee, Discussion paper on treatment of patients in persistent vegetative state, London: BMA, 1992.

[6] Institute of Medical Ethics Working Party on the Ethics of Prolonging Life and Assisting Death. Withdrawal of lifesupport from patients in a persistent vegetative state, Lancet 337 (1993), 96-98.

[7] BMA Medical Ethics Committee, BMA guidelines on treatment decisions for patients in a persistent vegetative state, London: BMA, 1994.

[8] N. Chomsky, Knowledge of Language Its Nature, Origins, and Use (Convergence), New York, Washington: Praeger Publishers, 1986.

[9] J.R. Cautela and A.J. Kearney, The Covert Conditioning Handbook, (Springer Series on Behavior Therapy and Behavioral Medicine) Vol. 17, Berlin, Springer, 1986.

[10] M. Kutas and S.A. Hillyard, Reading senseless sentences: brain potentials reflect semantic incongruity, Science 207 (1980), 203-205.

[11] S. Bentin, M. Kutas and S.A. Hillyard, Electrophysiological evidence for task effects on semantic priming in auditory word processing, Psychophysiology 30 (1993), 161-169.

[12] P.W. Schoenle and D. Schwall, Habituation des Blinkreflexes als Zeichen früher Lernvorgänge bei apallischen Patienten (Habituation of the blink reflex as index of early learning in patients in the vegetative state), in: Spektrum der Neurorehabilitation, K. von Wild, ed., Zuckschwerdt, München, 1993, pp. 158-163.

[13] J.T. Giacino, S. Ashwal, N. Childs, R. Cranford, B. Jennett, D.I. Katz, J.P. Kelly, J.H. Rosenberg, J. Whyte, R.D. Zafonte and N.D. Zasler, The minimally conscious state, Definition and diagnostic criteria, Neurology 58 (2002), 349-353.

[14] W. Witzke and P.W. Schoenle, Ereigniskorrelierte Potentiale als diagnostisches Mittel in der Neurologischen Führehabilitation (Event related potentials in the diagnostics of patients in neurological early rehabilitation), Neurol Rehabil 2 (1996), 68-80. 